Service social

\title{
Une nouvelle légitimité pour l'État social : par-delà le bien-être, le plaisir d'agir
}

\section{Daniel Tremblay}

Volume 52, numéro 1, 2006

URI : https://id.erudit.org/iderudit/015954ar

DOI : https://doi.org/10.7202/015954ar

Aller au sommaire du numéro

Éditeur(s)

École de service social de l'Université Laval

ISSN

1708-1734 (numérique)

Découvrir la revue

Citer cet article

Tremblay, D. (2006). Une nouvelle légitimité pour l'État social : par-delà le bien-être, le plaisir d'agir. Service social, 52(1), 47-64.

https://doi.org/10.7202/015954ar
Résumé de l'article

Du milieu des années 1970 jusqu'au début des années 1990, le ton des débats sur le rôle social de l'État était pessimiste (décennies de la " crise de l'État-providence »). S'appuyant sur les connaissances scientifiques pertinentes (principalement issues de la recherche évaluative), la thèse soutenue dans cet article est que le rôle de l'État est maintenant envisagé de manière plus positive, voire volontariste. Certains affirment que nous vivons présentement dans une "société active ". Il est suggéré ici que cette façon de concevoir le rôle de l'État dans les pays industrialisés correspond à l'émergence d'une « nouvelle légitimité " pour les politiques et les pratiques sociales. Le lien entre cette légitimité et le " bien-être " de la population est examiné dans quatre secteurs importants de l'intervention étatique : l'emploi, le revenu, l'éducation et le logement. 


\section{Une nouvelle légitimité pour l'État social : par-delà le bien-être, le plaisir d'agir}

Daniel TREMBLAY Professeur

Département de travail social et des sciences sociales Université du Québec en Outaouais

Du milieu des années 1970 jusqu'au début des années 1990, le ton des débats sur le rôle social de l'État était pessimiste (décennies de la « crise de l'État-providence »). S'appuyant sur les connaissances scientifiques pertinentes (principalement issues de la recherche évaluative), la thèse soutenue dans cet article est que le rôle de l'État est maintenant envisagé de manière plus positive, voire volontariste. Certains affirment que nous vivons présentement dans une « société active ». Il est suggéré ici que cette façon de concevoir le rôle de l'État dans les pays industrialisés correspond à l'émergence d'une « nouvelle légitimité » pour les politiques et les pratiques sociales. Le lien entre cette légitimité et le «bien-être » de la population est examiné dans quatre secteurs importants de l'intervention étatique : l'emploi, le revenu, l'éducation et le logement.

\section{Mots-clés : activation, État social, politique sociale, bien-être, légitimité, emploi, revenu, éducation, logement.}

From the mid 1970s to the beginning of the 1990s, the tone of the debates about the social role of the state was pessimistic («welfare state crisis» decades). Using the relevant scientific knowledge (especially evaluation research), the argument of this paper is that the role of the state is now discussed in more positive and voluntarist terms. Some say that we now live in an " active society ». It is suggested here that this way of viewing the social role of the state in industrialized countries corresponds to the emergence of a «new legitimacy » for social policies and practices. The relation between this legitimacy and the "well-being » of the population is studied in four important areas of state intervention, i.e., employment, income, education and housing conditions.

Keywords: Activation, Social State, Social Policy, Well-being, Legitimacy, Employment, Income, Education, Housing. 


\section{INTRODUCTION}

L'État s'affaire actuellement à se donner une «nouvelle légitimité » dont les conséquences sur les politiques et les pratiques sociales sont majeures. Le phénomène traverse l'ensemble du monde industrialisé. Il est apparu vers le milieu des années 1980 et a pris beaucoup d'ampleur depuis. Avant de chercher à reconnaître ses manifestations et à analyser ses enjeux dans quelques-uns des secteurs de l'intervention sociale, dressons une courte liste des notions que mobilise cette nouvelle légitimité. Cette expression, précisons-le d'abord, renvoie ici de façon assez classique à la « reconnaissance dont bénéficie [l']ordre politique » (Boudon, Besnard, Cherkaoui et Lécuyer, 2003, p. 132) de l'État social dans les diverses formes qu'il emprunte en cette ère de «post-providentialisme ». Selon une « architecture » (Jenson, 2004) qui est propre à chaque régime d'État social existant (Esping-Andersen, 1999), cette légitimité conjugue des notions maintenant familières à tous ceux et celles qui œuvrent dans le champ de l'intervention sociale : activation, citoyenneté active, participation citoyenne, communauté, appartenance, responsabilité, partenariat, entrepreneuriat, empowerment, résilience, autonomie, etc. Et l'on pourrait facilement enrichir cette liste de notions plus ou moins connexes comme celles de capital social, d'investissement social ou de patrimoine social. Toutes ces expressions, même si elles renvoient à des réalités très diverses, ont ceci de commun qu'elles sont aujourd'hui mises à contribution pour tenter de nommer les diverses figures de la nouvelle légitimité de l'État social.

II faut d'emblée souligner que ce discours, à l'opposé de celui qui dominait au cours de la majeure partie des années 1970 et 1980 , se veut positif et même productif. À la limite, on pourrait même le qualifier d'optimiste ou, de façon plus critique, de volontariste lorsqu'il attribue à l'État une capacité d'action dont la clairvoyance serait supérieure à celle dont sont capables les destinataires de son intervention. En ce sens, ce discours contraste avec le ton beaucoup plus négatif, déprimé et moins mobilisateur de cette époque, pourtant récente, où l'on dissertait sur la crise, le recul, le désengagement, la faillite ou la fin de l'État-providence (Beauchemin, Bourque et Duchastel, 1995; Bernier, 2003; Pierson, 1994).

Au-delà de la sémantique, on sait que le discours sur l'État social est traversé depuis trois décennies par des débats idéologiques qui ont été abondamment analysés et commentés (Tremblay, 2005). L'une des hypothèses examinées dans cet article est que ces débats, dont la pertinence n'est pour autant aucunement remise en cause ici, s'inscrivent dans un processus de mutation plus discret et néanmoins plus durable et profond. La nouvelle légitimité évoquée dans le titre de cet article est le produit de ce processus, un produit encore inachevé, mais qui est suffisamment développé pour être soumis à l'analyse. C'est en postulant l'existence de cette nouvelle légitimité que l'on peut se permettre de regrouper au sein du même champ sémantique des notions qui, autrement, sont souvent opposées l'une à l'autre. Par exemple, dans les débats entre les conceptions libérale et communautarienne de la citoyenneté, les notions d'autonomie et de communauté sont fréquemment mises en opposition (Berten, da Silveira et Pourtois, 1997; Etzioni, 1996). En les situant dans le même champ 
sémantique, on suggère qu'elles émanent au contraire du même terreau, celui de la nouvelle légitimité analysée dans cet article.

\section{L'« ÉVALUATION » DE L'ACTIVATION}

Pour ancrer l'analyse dans la réalité des politiques et des pratiques qui servent de cadre à l'intervention sociale, nous utiliserons les résultats d'une recension d'écrits scientifiques ayant pour objectif principal de dresser le bilan des connaissances actuelles sur les politiques et mesures publiques visant à rendre actifs les destinataires de l'intervention sociale ${ }^{1}$. À l'origine de ces politiques et mesures, on trouve généralement ce que le sociologue Anthony Giddens appelle un «État d'investissement social qui encouragerait l'initiative et la responsabilité individuelles plutôt que la passivité et la résignation »(2000, p. 67). C'est par « analogie avec le domaine des politiques de l'emploi », souligne pour sa part Simon Wuhl, que ces «nouvelles politiques mettent l'accent sur la solidarité active qui se substitue ainsi à la solidarité passive gérée mécaniquement par l'État-providence » (1996, p. 80). Dans les écrits recensés, il est en fait courant que soient appréhendées de façon simultanée les politiques qui cherchent, d'une part, à concilier la « protection sociale » et la « participation au marché du travail » et, d'autre part, à rendre actifs aussi bien les destinataires de l'intervention que les dispositifs d'intervention eux-mêmes (Barbier et LudwigMayerhofer, 2004; Halvorsen et Jensen, 2004).

Cette recherche, qui vise à faire la synthèse des publications scientifiques, met l'accent sur les efforts d'évaluation auxquels ont été soumises les politiques et mesures d'activation mises en place dans l'ensemble du monde industrialisé depuis le milieu de la décennie 1980 jusqu'à aujourd'hui. Quatre champs d'intervention, qui correspondent à ce que la littérature spécialisée appelle généralement les «conditions de vie » des destinataires, ont été retenus: l'emploi, le revenu, l'éducation et le logement. Cette recherche s'intéresse donc au vaste champ du « social » et aux efforts déployés par les États industrialisés au cours des quinze ou vingt dernières années pour activer leurs interventions dans ce domaine. De manière plus spécifique, cette recherche vise surtout à déterminer si ces efforts contribuent à améliorer le bien-être des destinataires de l'intervention. On reviendra, dans la prochaine section, sur cette notion de bien-être qui, il va de soi, occupe une place centrale dans toute intervention à visées sociales.

On pourrait disserter longtemps sur la stratégie de l'activation. Les écrits portant sur le sujet sont déjà nombreux, et cette stratégie traverse l'ensemble des changements apportés aux façons de faire de l'État social au cours des deux dernières décennies dans l'ensemble du monde industrialisé (Clasen et Clegg, 2003; Handler, 2003; Skevik, 2005). Elle a été modulée en fonction des divers régimes d'État social et il est clair qu'elle n'a pas échappé aux débats idéologiques entourant, par exemple, le rôle

1. Cette recherche a été financée par le Conseil québécois de la recherche sociale, maintenant fusionné au Fonds québécois de la recherche sur la société et la culture (projet RS 3255). Le rapport remis à l'organisme (Tremblay, Assogba, Boucher et Guindon, 2002) contient une description détaillée de la méthode utilisée. 
économique et social de l'État ou les rapports entre ce dernier, les organisations de la société civile, les communautés, les familles et les individus (Esping-Andersen, 1999; Esping-Andersen, Gallie, Hemerijck et Myles, 2002; Gilbert, 2002).

C'est dans le secteur de l'emploi que le phénomène s'est manifesté de la manière la plus explicite et la plus systématique, mais la stratégie de l'activation a également été administrée dans pratiquement tous les domaines où intervient l'État social. On aura l'occasion, dans la suite de cet article, d'en citer plusieurs exemples et d'examiner l'état des connaissances au sujet des effets de cette stratégie sur le bien-être de ses destinataires. Toutes les politiques ou mesures publiques d'activation, en dépit de leur diversité, ont en commun de miser sur la dynamisation (mobilisation, stimulation, incitation, etc.) des destinataires de l'intervention, qu'il s'agisse d'acteurs individuels ou d'acteurs collectifs ou encore de milieux de vie (ex. : vie de quartier). L'activation présuppose en effet que ces destinataires souffrent de ce que l'on pourrait appeler un " déficit de dynamisme » (manque de volonté, de tonus, d'estime de soi, d'ambition, etc.), et la stratégie privilégiée consiste à essayer de combler ce déficit. Ce mode d'intervention tranche avec celui que privilégiait souvent le providentialisme. Rien n'est moins passif, en fait, que la nouvelle légitimité de l'État social. Au contraire, c'est sur un mode résolument productiviste que celle-ci se présente et qu'elle confère à l'État social actuel un visage et un rôle bien différents de ceux qu'avait l'État-providence jusqu'à récemment. C'est dans ce contexte que l'on a par exemple pu observer, au cours des quinze dernières années, une imbrication croissante des politiques d'emploi et de plusieurs politiques sociales (Barbier et Ludwig-Mayerhofer, 2004). Le phénomène a pris des formes assez différentes d'un pays à l'autre et surtout d'un régime d'État social à l'autre (ex. : l'opposition entre la logique de l'insertion et celle du workfare), mais la tendance est universelle, du moins dans le périmètre de l'Organisation de coopération et de développement économiques (OCDE). Pour les partisans du libéralisme économique, nul n'est mieux placé que l'individu lui-même pour s'« aider » (d'où l'acharnement à dénoncer la « dépendance » qu'engendre à leurs yeux le providentialisme). Par ailleurs, même les plus chauds partisans de l'interventionnisme étatique n'osent plus aujourd'hui faire référence à une «aide » sans contrepartie (Dufour, Boismenu et Noël, 2003) ${ }^{2}$.

\section{LE BIEN-ÊTRE : UN ÉTAT, UNE SENSATION, UNE AMBITION?}

La notion de bien-être, qui loge au cœur du providentialisme, reste bien sûr centrale dans tout discours visant à légitimer l'intervention sociale. Cela est si vrai qu'on s'est relativement peu interrogé sur cette question pendant de nombreuses années. Que l'État-providence ait pour fondement l'amélioration du bien-être de ses destinataires relève apparemment de l'évidence (Boychuk, 2004; Jenson, 2004). On s'est bien efforcé, vers la seconde moitié des années 1960 et la première moitié des années 1970, de préciser la notion de bien-être et de la rendre plus observable, concrète et mesurable. Cela a donné lieu à ce qu'il est convenu d'appeler le «mouvement des

2. Certains auteurs parlent d'« activation positive » pour décrire les mesures incitatives et d'« activation négative » pour désigner les « politiques contraignantes » (Taylor-Gooby, 2004). 
indicateurs sociaux ». À partir du milieu des années 1970, au moment où le providentialisme commençait à subir les assauts de l'idéologie néolibérale, ce « mouvement » s'est un peu essoufflé (Perret, 2002).

Depuis le début des années 1990, la question du bien-être connaît cependant un important regain d'intérêt. Les travaux de réflexion et de recherche sur cette question se sont multipliés au cours des quinze dernières années. Que l'on pense, par exemple, aux travaux de l'économiste Amartya Sen (1993) sur le sujet, lesquels ont fortement influencé le Programme des Nations unies pour le développement (PNUD) qui, par la publication de ses rapports annuels, a vivement contribué à élargir et soutenir l'intérêt pour cette notion depuis le début des années 1990 (Destremau et Salama, 2002). Même la Banque mondiale et l'OCDE, depuis quelques années, ont joint les rangs de cette nouvelle mobilisation en faveur du bien-être (Gadrey et Jany-Catrice, 2003; Healy et Côté, 2001).

À ce jour, aucune définition du bien-être n'a fait l'unanimité, mais on s'emploie tout de même avec beaucoup d'énergie à mieux cerner cette notion, à en approfondir le sens, à en soupeser les aspects objectifs et subjectifs, à en départager les dimensions individuelle, familiale, communautaire et publique, à en faire ressortir les éléments de nature plus quantitative et plus qualitative, à réfléchir sur l'importance respective des ressources, des capacités et des attentes, à proposer des systèmes d'indicateurs socioéconomiques pour «mesurer » ce bien-être et éventuellement en suivre la progression (Gadrey et Jany-Catrice, 2003; Osberg et Sharpe, 2002; Prescott-Allen, 2003; Ringen, 1997). II faut remonter aux années 1960 pour trouver un foisonnement comparable de travaux sur la question du bien-être et sur les thèmes qui lui sont connexes.

Le bien-être, comme notion et comme ambition, est donc loin d'avoir abandonné tout le terrain aux thèmes de la croissance et de la richesse dont on parle également beaucoup depuis quelques années. S'il est vrai, en d'autres mots, que le néolibéralisme s'est taillé la part du lion dans le paysage idéologique des deux dernières décennies, les traces du providentialisme ne sont pas pour autant complètement disparues. C'est plutôt à une transformation en profondeur de l'État social que l'on assiste, celui-ci s'employant à se doter d'une nouvelle légitimité.

Dans le présent article, le concept de bien-être correspond à ce que Robert PrescottAllen appelle le « bien-être humain », notion qu'il définit comme suit : "Le bien-être humain est un état dans lequel tous les membres d'une société sont capables de déterminer leurs besoins et de les satisfaire, et ont à leur disposition un vaste éventail de choix pour réaliser tout leur potentiel » (2003, p. 13). Les politiques et mesures d'activation sur lesquelles portent les écrits recensés dans la prochaine section visent en effet, de différentes façons, à faire en sorte que les destinataires de l'intervention soient mieux en mesure de « déterminer et satisfaire leurs besoins » et de « réaliser 
tout leur potentiel ». L'activation, comme stratégie d'intervention, valorise d'ailleurs grandement cette idée de « potentiel ${ }^{3} »$.

\section{ACTIVATION ET BIEN-ÊTRE : ANALYSE DE LA DOCUMENTATION SCIENTIFIQUE}

La recherche sur laquelle s'appuie cet article a permis de relever plus de 200 publications scientifiques, en langue française ou anglaise, parues depuis le milieu des années 1980 et dans lesquelles sont mises en relation les notions d'activation et de bien-être (Tremblay, Assogba, Boucher et Guindon, 2002). On ne peut, bien sûr, exposer ici l'ensemble des résultats obtenus. Ces derniers seront présentés sous forme de synthèse, une sous-section étant consacrée à chacune des « conditions de vie » mentionnées plus haut (emploi, revenu, éducation et logement).

\section{L'EMPLOI}

On sait que ce champ d'intervention est celui dans lequel les politiques et les mesures d'activation ont été les plus nombreuses. Le discours sur la « société active » de l'OCDE (Walters, 1997) se concentrait, à l'origine, dans ce domaine (ex. : qualification de la main-d'œuvre, création d'emplois, insertion professionnelle, soutien à l'entrepreneuriat). Il s'est sensiblement élargi au cours de la décennie 1990. Avant d'examiner la littérature concernée, il convient cependant de préciser que, même si le discours sur la " société active » a eu un grand retentissement au cours des quinze dernières années, le niveau des investissements publics en faveur de l'activation dans le domaine de l'emploi est loin d'avoir connu une progression d'une importance comparable. C'est du moins ce qui se dégage des travaux portant sur l'évolution des dépenses d'activation en emploi dans les pays membres de l'OCDE (1998). Le Canada, comme l'indiquent des travaux récents, a d'ailleurs emprunté la même voie. Au moment où y progressait le discours sur l'activation, la part relative des « dépenses sociales actives » canadiennes dans le domaine de l'emploi avait tendance à régresser (Boychuk, 2004). Cela dit, il reste que c'est au sujet de l'activation des politiques et mesures liées à l'emploi que les écrits scientifiques nous fournissent le plus d'information.

Pour aller à l'essentiel, il en ressort globalement que la question du bien-être y est peu traitée. Ces politiques et mesures sont généralement évaluées en fonction de leur impact sur la lutte contre le chômage et sur le développement de l'emploi (Fay, 1996). Même sur ces questions, les recherches existantes sont assez peu explicites et rarement concluantes. L'activation, dit-on, donnerait souvent des « résultats équivoques » (Barbier et Ludwig-Mayerhofer, 2004, p. 428). Après avoir prôné la stratégie de l'activation dans le domaine de l'emploi avec conviction depuis de nombreuses années, l'OCDE admettait à l'issue des années 1990 que l'on en connaît encore mal les effets (Martin, 2000). Comme le soulignent des auteurs québécois après avoir comparé des pratiques en ce domaine dans plusieurs pays européens et nord-

3. Le Petit Robert précise que le substantif «potentiel », au cours du $X X^{e}$ siècle, a pris le sens de « capacité d'action, de production ». 
américains, « le bien-fondé intrinsèque des mesures actives n'a jamais réellement été démontré » (Dufour, Boismenu et Noël, 2003, p. 126; voir aussi Esping-Andersen, 1999, p. 183).

Sous l'angle de leur contribution au bien-être, l'apport des politiques et mesures actives en emploi est encore moins clair. C'est à peine si l'on s'est efforcé de le vérifier et encore moins de le mesurer. S'appuyant sur une synthèse des « études d'évaluation » des politiques et mesures appliquées dans les pays industrialisés, un document publié par l'OCDE conclut par exemple qu'« [i]l n'y a guère de données disponibles sur les retombées sociales possibles de la participation aux programmes, par exemple en termes de recul de la délinquance ou de la toxicomanie, ou d'amélioration de la santé » (Martin, 2000, p. 98; voir aussi Gilbert et Van Voorhis, 2001, p. 303).

Toujours du point de vue de leur incidence sur le bien-être de leurs destinataires, les politiques et mesures actives dans le domaine de l'emploi semblent donner des résultats ambivalents même lorsqu'elles sont utilisées dans un contexte qui, a priori, leur est favorable. Des chercheurs en sont par exemple arrivés à la conclusion que l'expérience suédoise, pourtant considérée comme un « modèle » dans ce domaine et qui reste associée au régime social-démocrate bien plus qu'au régime libéral en matière de politique sociale, aurait «paradoxalement » eu tendance à affecter à la baisse l'autonomie, le moral et la motivation des destinataires de l'activation lorsque la Suède a dû faire face à une brutale hausse du chômage au début des années 1990 (Hallsten, Grossi et Westerlund, 1999). Les auteurs concluent que les mesures d'activation, à elles seules, ne suffisent pas et qu'elles doivent être constamment renouvelées pour ne pas donner à leurs destinataires l'impression qu'ils peuvent compter sans réserve sur ces mesures. L'activation, en d'autres termes, ne serait pas à l'abri du «syndrome de la dépendance » que dénoncent les critiques du providentialisme. Dans les pays scandinaves, les mesures d'activation qui semblent avoir l'effet le plus positif sur la « qualité de vie » de leurs destinataires sont celles qui font l'objet d'un suivi régulier, direct et individualisé (Halvorsen et Jensen, 2004). Selon certaines évaluations récentes, la valorisation de l'activation, qui a contribué à ce que la littérature européenne appelle le «miracle hollandais », aurait eu pour effet d'accentuer les sentiments d'isolement et d'inutilité sociale éprouvés par ceux et celles que ce « miracle » a oubliés (Oorschot, 2004).

Le terme «ambivalence » utilisé dans le paragraphe précédent s'applique aux effets de la plupart des mesures actives dans le domaine de l'emploi lorsqu'elles sont examinées sous l'angle du bien-être. Dans un pays comme la Suède, on estime par exemple que les femmes ont sans doute tiré avantage de l'existence de ces mesures, mais au prix d'un certain paternalisme et d'une dépendance, encore une fois, à l'égard de l'État (Baker, 1999). Aux États-Unis et dans plusieurs pays de culture anglosaxonne, où les approches retenues sont en général diamétralement opposées, sur le plan idéologique, à celles que l'on retrouve dans les pays scandinaves, ces politiques et mesures ont fait l'objet de nombreuses évaluations. La question du bien-être y tient une place assez marginale, mais les études qui s'y sont attardées démontrent que les résultats sont ou impossibles à démontrer ou fort modestes (Baker et North, 1999; 
Baker et Tippin, 1999). Cette situation a donc pour effet de laisser une large part à l'interprétation dans le discours qui se tient au sujet de l'activation dans le secteur de l'emploi.

Avant de passer au thème du revenu, il convient de souligner que ce n'est pas un hasard si la situation des femmes est souvent abordée dans les écrits recensés. L'arrivée massive et rapide des femmes sur le marché du travail est un phénomène qui se heurte à une réalité qui reste nettement différenciée selon les sexes en matière de responsabilités familiales. Or, les politiques et mesures actives ont largement été utilisées par les pouvoirs publics pour tenter de résoudre ou du moins d'atténuer l'impact de cet enjeu (Skevik, 2005; Walker et Wiseman, 2003). Le moins que l'on puisse dire, c'est que les résultats obtenus à ce jour n'ont rien de bien spectaculaire. Cela dit, des recherches indiquent que les femmes en difficulté (ex. : faibles revenus, monoparentalité) qui sont exposées à ce type d'interventions, particulièrement lorsqu'elles n'y sont pas forcées et que leurs enfants n'ont pas à en faire les frais, ont tendance à apprécier que l'on cherche à leur venir en aide (Hagen et Davis, 1996). Leur « estime de soi » s'en trouve améliorée, elles se sentent plus « autonomes » et elles ont l'impression d'offrir ainsi à leurs enfants un « modèle de vie plus positif ». Comme on peut le constater, le regard que porte la société sur leur situation semble jouer un rôle important dans le jugement qu'elles portent à leur tour sur les mesures d'activation qui leur sont proposées.

\section{LE REVENU}

Les « conditions de vie » liées au thème du revenu sont souvent liées également à la question de l'emploi. Même si tous les individus à faible revenu ne sont pas forcément sans emploi, il reste que c'est fréquemment le cas. Une bonne partie des mesures d'activation mises en place dans les pays industrialisés ciblent donc les plus démunis. Ces mesures ont généralement pour objectif d'aider leurs destinataires à s'insérer ou se réinsérer dans la société, de préférence par l'intermédiaire de l'insertion professionnelle. Ces destinataires sont souvent jeunes ou de sexe féminin, et les mesures dont ces personnes sont l'objet ont notamment pour fonction de renforcer leur «autonomie », leur « confiance en soi », leur «sens des responsabilités », leurs « compétences parentales ».

La clientèle de ces politiques et mesures d'activation est celle qui a été la plus directement affectée par les «réformes » réalisées dans le champ de l'assistance publique au cours des deux dernières décennies. Les problèmes psychosociaux et de santé auxquels sont exposés ces individus sont nombreux et variés: grossesse précoce, violence et abus envers les enfants, prostitution, alcoolisme et toxicomanie, transmission intergénérationnelle de la dépendance, décrochage scolaire, etc.

Dans la mesure où ce segment de population est largement perçu par l'opinion publique comme étant en difficulté, il semble relativement facile de justifier les politiques et mesures d'activation qui lui sont destinées. Des études indiquent d'ailleurs qu'une bonne partie de la population, du moins aux États-Unis, semble adhérer à l'idée 
que l'existence de ces politiques contribuerait à briser le moule de la « dépendance » dans lequel, estime-t-on, serait confinée la «clientèle de l'assistance publique » (Besley et Coate, 1992; Moffitt, 1992). Au-delà de cette appréciation à tout le moins subjective, on en sait toujours peu sur les effets qu'ont ces politiques et mesures sur le bien-être de leurs destinataires. On s'est davantage employé à étudier les effets de la « dépendance » que créeraient ces programmes pour en arriver à la conclusion assez prévisible qu'elle affecte le bien-être (faible estime de soi, sentiment d'impuissance, détresse psychologique...) des femmes concernées et de leurs enfants (Elliott, 1996; Ensminger, 1995).

Les quelques études d'évaluation qui se sont intéressées à la question du bien-être des jeunes mères en difficulté socioéconomique et de leurs enfants ont tendance à conclure que les politiques et mesures d'activation donnent des résultats mitigés. Ces études ont par exemple permis de constater que l'activation ne semble pas nuire au bien-être de ces mères et de leurs enfants (Aber, Brooks-Gunn et Maynard, 1995; McClintock et Lowe, 2001). Faute de pouvoir démontrer qu'elles contribuent au mieuxêtre de leurs destinataires, on constate donc qu'elles ne sont généralement pas nocives. Cette conclusion ambiguë permet aussi bien aux tenants de l'activation de faire valoir les mérites de cette approche qu'à leurs opposants d'en souligner l'inefficacité. Des auteurs soutiennent que l'on devrait mettre fin à cette ambiguïté et faire preuve de plus de transparence, en ciblant explicitement l'amélioration du bienêtre des enfants (childfare) si c'est ce qui est vraiment visé, au lieu de se servir de ce prétexte pour chercher à justifier l'approche du workfare (Baratz et White, 1996).

Pour conclure sur le thème du revenu, il convient de souligner que les résultats discutés dans cette section découlent tous de travaux réalisés aux États-Unis. Généralement centrées sur des individus, les mesures d'activation y font l'objet de débats assez étroits. Faut-il, par exemple, utiliser la contrainte ou plutôt la persuasion en ce qui a trait à la participation de la clientèle visée à ces mesures d'activation? Des questions du genre, les écrits recensés en posent souvent. Le thème du bien-être reste dans l'ombre de ce questionnement. Les écrits teintés de l'idéologie libérale y répondent sans difficulté, mais aussi sans grand souci de rigueur. Les études qui s'appuient sur une méthodologie plus exigeante tirent pour leur part des conclusions souvent ambiguës ou du moins fort nuancées.

\section{L'ÉDUCATION}

Dans le domaine de l'éducation, de nombreuses politiques et mesures d'activation ont été mises en place dans le monde industrialisé au cours des quinze ou vingt dernières années. Ces programmes et mesures, qui visent principalement les enfants et les jeunes mais aussi leurs parents, veulent « éduquer à la citoyenneté », promouvoir une « citoyenneté active », faire acquérir des «compétences sociales », encourager les « partenariats parents-écoles », stimuler l'« intervention précoce et bigénérationnelle », etc. Ce sont donc des politiques et des mesures qui, tout comme c'est le cas en matière d'emploi et de revenu, privilégient comme mode d'intervention la dynamisation de leurs destinataires. 
Les écrits consacrés à l'impact de ces politiques et mesures sur le bien-être des enfants, des jeunes et de leurs parents sont moins nombreux que dans les domaines de l'emploi et du revenu. La littérature recensée réitère les mêmes avantages qui sont prêtés à l'activation dans les autres domaines d'intervention, mais généralement sans chercher à vérifier si cette activation contribue effectivement au mieux-être de ses destinataires. On s'y emploie, par exemple, à prôner dans une optique de participation active le renforcement et l'assouplissement des interactions entre les parents et les établissements scolaires ou les professionnels de l'enseignement. Cette approche, soutient-on, favoriserait la réussite scolaire des enfants et contribuerait à lutter contre l'absentéisme et l'abandon scolaires. On fournit par ailleurs très peu d'illustrations permettant de constater que le bien-être des enfants s'améliore effectivement sous l'effet de ces stratégies d'intervention (Birenbaum-Carmeli, 1999; McKenna et Willms, 1998; St. John-Brooks et Kelley-Lainé, 1997).

Observant que les enfants et les jeunes, en matière d'éducation, sont souvent cantonnés dans des rôles relativement passifs, on s'emploie depuis quelques années à stimuler leur participation active. Sur le plan scolaire, bien sûr, mais aussi dans d'autres champs d'action comme la promotion de la santé (Winter, Baerveldt et Kooistra, 1999), l'utilisation des médias (Buckingham, 1997) ou l'éducation à la citoyenneté (McAndrew, Tessier et Bourgeault, 1997). Ce dernier thème, en particulier, connaît une grande popularité depuis quelque temps. Les écrits recensés ne comportent pas, à proprement parler, d'études d'évaluation permettant de conclure que l'éducation à la citoyenneté contribue effectivement au bien-être de ses destinataires ou même à celui de la communauté. On soutient qu'elle est de nature à renforcer la cohésion sociale, à stimuler l'entraide et à faciliter les contacts entre les cultures, mais cette démonstration reste à faire. Certains auteurs estiment même que le fait d'inviter les jeunes à faire preuve d'une citoyenneté active, sans agir en parallèle sur les conditions sociales et économiques dans lesquelles devrait s'exercer cette citoyenneté, équivaut plus à renforcer le conformisme qu'à dynamiser la participation de ces jeunes à la vie collective (France, 1998).

L'activation dans le domaine de l'éducation ne se pratique pas qu'à l'intérieur des systèmes scolaires. II est courant, depuis quelques décennies, de privilégier aussi ce type d'approche dans les interventions visant les enfants d'âge préscolaire. C'est ce que l'on appelle, dans les écrits recensés, les programmes d'intervention précoce et, parfois, bigénérationnelle. À ce jour, ces approches ont été utilisées de façon assez large dans certains pays européens et de façon plus ciblée en Amérique du Nord. Comme c'est le cas pour la majorité des politiques et mesures visées par cette recherche, les expériences et pratiques américaines ont été plus souvent évaluées que celles des autres pays. II se dégage de ces travaux, surtout ceux effectués dans une optique comparative, que les mesures les plus efficaces sont celles qui s'inscrivent dans un cadre plus large, qui intègre par exemple ces programmes dans une politique familiale nationale ou une stratégie de lutte contre les inégalités socioéconomiques (McGovern, 1998; Spence Boocock, 1995). Pour ce qui est, plus spécifiquement, des programmes d'intervention précoce ou bigénérationnelle, leur utilité serait plus évidente du point de vue cognitif que social. Les évaluations existantes montrent en 
effet que ces programmes semblent améliorer les chances de réussite scolaire des enfants venant de milieux défavorisés (Barnett, 1995; Zaslow, Oldham, Moore et Magenheim, 1998). Les mères de ces enfants, dans une moindre mesure, en tireraient également avantage sur le plan des «compétences parentales » et du « capital culturel » (Gustafsson et Stafford, 1995; Orthner et Randolph, 1999; St. Pierre, Layzer et Barnes, 1995).

\section{LE LOGEMENT}

En matière de logement, les politiques et mesures d'activation peuvent prendre plusieurs formes. À la différence des interventions «traditionnelles » dans le domaine, elles ne visent généralement pas à mettre un toit à la disposition de ceux et celles qui n'en ont pas, à rendre le logement plus accessible ou à améliorer la salubrité d'un habitat. Elles visent plutôt, par exemple, à revitaliser un environnement social (quartier, communauté rurale...), à faciliter l'intégration ou l'insertion de personnes en déficit d'autonomie, à améliorer les relations entre propriétaires et locataires, à stimuler la « participation citoyenne dans un milieu de vie », à « territorialiser les politiques », etc. Ce sont des interventions qui, comme celles qui ont été évoquées dans les sections précédentes, s'inscrivent dans une optique de dynamisation.

Les écrits qui s'intéressent à de telles mesures sont nettement moins nombreux que ceux qui portent sur l'emploi ou le revenu. L'attention qu'on y accorde à la question du bien-être des destinataires de l'intervention est également encore plus limitée. Si l'on prône, par exemple, la « revitalisation sociale » d'un milieu, c'est avec l'idée d'améliorer la « qualité de vie » des gens qui l'habitent (Brink, 1996), mais peu de travaux ont permis de vérifier dans quelle mesure cet objectif est atteint. De même, si l'on favorise dans les interventions en faveur des personnes en difficulté (problèmes socio-affectifs ou de santé physique, mentale, etc.) des formules aussi « normalisantes » et participatives que possible, c'est au nom de leur « estime de soi » ainsi que du respect de «leur autonomie, de leur identité et de leur intimité ». Cependant, les écrits recensés sont peu explicites sur les résultats obtenus. Certains auteurs soutiennent même qu'il faudrait faire preuve de plus de patience avant de chercher à tirer des conclusions au sujet de ces interventions (Ridgway, Simpson, Wittman et Wheeler, 1998; Ware, 1999).

Une partie des écrits recensés mettent l'accent sur l'engagement que manifestent ou devraient manifester les acteurs sociaux en faveur de leur « milieu de vie » (Donzelot et Estèbe, 1994; Kleinman et Whitehead, 1999; Weicher, 1996). II se dégage de ces écrits que les personnes qui éprouvent un sentiment d'appartenance à leur milieu ont davantage tendance à contribuer au renforcement de la cohésion sociale, à la lutte contre la criminalité, au développement d'un meilleur voisinage ainsi qu'à l'amélioration de leur cadre de vie matériel et social. En facilitant, par exemple, l'accès à la « propriété », on contribuerait aussi au mieux-être des destinataires de l'intervention. Les recherches effectuées à ce jour suggèrent toutefois que les individus et les familles dont le sort s'améliore ont tendance à vouloir rehausser encore davantage leurs conditions de vie en se déplaçant vers des environnements plus favorisés, ce qui ne 
contribue guère à revitaliser durablement les environnements plus précaires (Atkinson et Kintrea, 2000). Par ailleurs, la «cohabitation» de familles qui vivent dans des conditions difficiles mais similaires (Hasell et Scanzoni, 1997), laquelle « cohabitation » est parfois prônée parce qu'elle stimulerait l'« entraide » au sein de ces communautés, présente bien sûr l'inconvénient d'accentuer le cloisonnement et la stigmatisation de ces communautés.

On peut en tirer la conclusion que les mesures d'activation expérimentées dans le domaine du logement donnent rarement des résultats monovalents. Le contexte économique et sociologique dans lequel prennent place ces mesures joue un rôle déterminant, de sorte qu'il est difficile d'attribuer aux seules politiques et mesures relatives au logement des effets bénéfiques ou délétères, du moins sous l'angle du bien-être. Comme le soulignent certains auteurs, l'amélioration de la qualité de vie par le biais du logement passe forcément aussi par l'éducation (information, sensibilisation, etc.) et par l'amélioration des conditions de vie socioéconomiques des destinataires de l'intervention (Somerville, 1998; Somerville et Steele, 1999).

\section{CONCLUSION : LE PLAISIR D'AGIR}

Cette synthèse des écrits montre de façon assez nette que, contrairement à l'optique providentialiste dans laquelle prédominent les notions de protection et de sécurité, la nouvelle légitimité de l'État social se caractérise par une assimilation, sous diverses variantes (autonomie, responsabilité, citoyenneté active, empowerment, promotion, résilience, etc.), des notions d'activité et de bien-être. On garde l'impression que les risques contre lesquels l'État-providence protégeait ses « clients » (maladie, chômage, pauvreté, criminalité, vieillesse, etc.) sont désormais censés se dissoudre dans l'activité. La nouvelle légitimité de l'État social, à la lumière des évaluations auxquelles elle a été soumise jusqu'à présent, semble dispenser ce dernier d'avoir à justifier son existence, comme devait constamment le faire l'État-providence à l'époque où on le disait « en crise ». C'est apparemment de manière plus optimiste que l'État social peut envisager son avenir, lequel passe maintenant par les notions d'activation, d'autonomie, de partenariat, d'investissement social, d'entrepreneuriat, de capital social, etc. Ces notions, par leur association et leur complémentarité, semblent conférer à l'État social une légitimité renouvelée, post-providentialiste, mais aussi volontariste et productiviste.

Par ailleurs, on peut penser que l'État social ne pourrait agir de la sorte s'il ne s'adressait à des sujets (s'agit-il toujours de «clients »?) qui aspirent de leur côté à l'autonomie, à la responsabilisation, au partenariat, à la citoyenneté active, à l'entrepreneuriat, à l'insertion, etc. Dans la mesure où ces «nouvelles compétences » ont de l'attrait pour ces sujets, ils n'ont pas à s'interroger sur leur bien-être, puisque ce dernier est à leur portée et que l'État social n'a plus d'autre rôle, dorénavant, que de les « aider » à réaliser leur « potentiel ». C'est en s'occupant qu'ils se sentiront mieux, et non en exigeant que l'on s'occupe d'eux. 
La notion de «potentiel », comme nous l'avons vu plus haut en définissant le concept de « bien-être », est aujourd'hui étroitement associée à ce dernier. II se peut, et ce n'est ici qu'une hypothèse, que l'« amalgame du potentiel et du bien-être » explique en partie notre relative méconnaissance de l'impact que l'activation a sur le bien-être. II y a en effet, dans l'idée même de «potentiel », la présupposition d'une capacité qui existe chez les destinataires de l'intervention, mais à l'état virtuel. Comment, par conséquent, la cerner, l'observer, la mesurer, l'évaluer? Autant le défi est considérable sur le plan méthodologique, autant la tentation semble forte, sur le plan idéologique, de ne pas consacrer trop d'énergie à faire la preuve de ce qui semble relever pour plusieurs du simple «bon sens ». Après tout, comme le soulignait Jean-Claude Barbier il y a quelques années, il paraît aller de soi « que les politiques actives et l'activation sont meilleures, par principe, que les politiques passives » (1996, p. 9).

Ayant pris ses distances avec la logique de la dépendance, de la passivité et du clientélisme qui, a-t-on souvent dit au cours des années 1980 et 1990, caractériserait le providentialisme, l'État social tend maintenant à justifier son existence en offrant à ses destinataires la perspective du « plaisir d'agir ». II s'est en effet instauré, au cours des dernières années, un nouveau type de rapport entre l'État social et ses destinataires (Cruikshank, 1999; Dean, 1999; Rose, 1999). Au-delà des droits sociaux et économiques, des transferts financiers, des mesures fiscales et des services publics (santé, adaptation sociale, éducation, etc.) à travers lesquels s'incarne l'Étatprovidence, c'est sur un autre plan, celui des perceptions, attitudes, compétences et comportements des sujets, qu'agit la nouvelle légitimité de l'État social. C'est dans ce cadre que l'activité semble maintenant devenue, en soi, une source de bien-être.

Les options précédentes, comme s'affaire à le démontrer l'OCDE depuis le début des années 1990, ont toutes été disqualifiées au profit du principe de la « société active » (Larsen, 2001; Tremblay, 2005; Walters, 1997). Après s'être d'abord imposé dans le secteur de l'emploi (Martin, 2000), où son intérêt sur le plan économique était plus évident et perçu comme plus pressant, l'attrait de ce principe n'a plus de frontières. On lui attribue d'innombrables vertus dans tous les domaines où se manifeste la nouvelle légitimité de l'État social et où s'établit un lien entre ce dernier et ses « partenaires » (Caddy et Vergez, 2001). C'est par exemple le cas en matière d'éducation, de formation et de cohésion sociale (Healy et Côté, 2001; St. John-Brooks et KelleyLainé, 1997), de logement et d'intégration sociale (Kirwan et MacFarlane, 1996), de vieillissement et de retraite (Shigehara, 1998). Par ailleurs, force est de constater qu'aucune des publications de l'OCDE n'établit, sur la base d'évaluations rigoureuses ou même d'indicateurs faisant l'objet d'un consensus minimal, que cette nouvelle légitimité contribue effectivement au mieux-être des sujets qu'elle mobilise, si ce n'est en distillant ce « plaisir d'agir » auquel ils sont conviés.

On a assisté, au cours des deux dernières décennies, à une transformation profonde de l'État social. L'« activité » fait aujourd'hui l'unanimité. Les débats sur le désengagement de l'État-providence, débats à forte teneur idéologique (arguments néolibéraux contre arguments sociaux-démocrates ou communautariens), ont largement occulté cette transformation, si bien qu'il semble devenu inutile de s'interroger sur 
l'apport des politiques et mesures d'activation au mieux-être de la population. Tout se passe comme si l'« activité » n'avait pas à convaincre qu'elle est un vecteur de bienêtre, puisque la nouvelle légitimité de l'État social donne désormais à la notion même de bien-être une signification différente de celle qu'elle avait aux « beaux jours » du providentialisme.

\section{RÉFÉRENCES BIBLIOGRAPHIQUES}

Aber, L., J. BRoOKS-GunN et R. MAYNARD (1995). «Effects of welfare reform on teenage parents and their children », Future of Children, vol. 5, $n^{\circ} 2$, p. 53-71.

ATKINSON, R., et K. KINTREA (2000). «Owner-occupation, social mix and neighbourhood impacts », Policy and Politics, vol. 28, $\mathrm{n}^{\circ}$ 1, p. 93-108.

BAKER, D., et K. NORTH (1999). «Does employment improve the health of lone mothers? », Social Science and Medicine, vol. 49, $\mathrm{n}^{\circ}$ 1, p. 121-131.

BAKER, M., et D. TIPPIN (1999). Poverty, Social Assistance, and the Employability of Mothers: Restructuring Welfare States, Toronto, University of Toronto Press.

BAKER, S. (1999). «Risking difference: Reconceptualizing the boundaries between the public and private spheres », dans S. BAKER et A. VAN DOORNE-HUISKES (dir.), Women and Public Policy: The Shifting Boundaries between the Public and Private Spheres, Aldershot, Ashgate, p. 3-31.

BARATZ, M., et S. WhITE (1996). «Childfare: A new direction for welfare reform », Urban Studies, vol. 33, n 10, p. 1935-1944.

BARBIER, J.-C. (1996). «Comparer insertion et workfare? », Revue française des affaires sociales, vol. $50, n^{\circ} 4$, p. 7-26.

BARBIER, J.-C., et W. LUDWIG-MAYERHOFER (2004). «Introduction: The many worlds of activation », European Societies, vol. 6, $\mathrm{n}^{\circ} 4$, p. 423-436.

BARNETT, S. (1995). « Long-term effects of early childhood programs on cognitive and school outcomes », Future of Children, vol. $5, \mathrm{n}^{\circ} 3$, p. 25-50.

BeAuchemin, J., G. Bourque et J. Duchastel (1995). « Du providentialisme au néolibéralisme : de Marsh à Axworthy. Un nouveau discours de légitimation de la régulation sociale », Cahiers de recherche sociologique, vol. 24, p. 15-47.

Berten, A., P. da Silveira et H. Pourtois (dir.) (1997). Libéraux et communautariens, Paris, Presses universitaires de France.

BESLEY, T., et S. COATE (1992). « Understanding welfare stigma: Taxpayer resentment and statistical discrimination », Journal of Public Economics, vol. 48, $n^{\circ} 2$, p. 165-183.

BiREnBAUM-CARMELI, D. (1999). « Parents who get what they want: On the empowerment of the powerful », The Sociological Review, vol. 47, $\mathrm{n}^{\circ}$ 1, p. 62-90.

Boudon, R., P. Besnard, M. Cherkaoul et B.-P. LÉCuyer (2003). Dictionnaire de sociologie, Paris, Larousse. 
Boychuk, G. (2004). The Canadian Social Model: The Logics of Policy Development, Ottawa, Canadian Policy Research Networks.

BRINK, S. (1996). «Revitalisation sociale et qualité du cadre de vie », dans R. KIRWIN et L. MACFARLANE (dir.), Stratégies pour le logement et l'intégration sociale dans les villes, Paris, OCDE, p. 73-162.

BuCKINGHAM, D. (1997). « The making of citizens: Pedagogy and address in children's television news », Journal of Educational Media, vol. 23, $n^{\text {os }} 2-3$, p. 119-139.

CAdDY, J., et C. VeRgez (2001). Des citoyens partenaires: information, consultation et participation à la formulation des politiques publiques, Paris, OCDE.

Clasen, J., et D. ClegG (2003). « Unemployment protection and labour market reform in France and Great Britain in the 1990s: Solidarity versus activation? », Journal of Social Policy, vol. 32, $n^{0} 3$, p. 361-381.

Cruikshank, B. (1999). The Will to Empower: Democratic Citizens and Other Subjects, Ithaca, Cornell University Press.

DEAN, M. (1999). Governmentality: Power and Rule in Modern Society, Londres, Sage Publications.

Destremau, B., et P. Salama (2002). Mesures et démesure de la pauvreté, Paris, Presses universitaires de France.

DONZELOT, J., et P. ESTĖBE (1994). L'État animateur : essai sur la politique de la ville, Paris, Esprit.

Dufour, P., G. BoISMENu et A. NoËL (2003). L'aide au conditionnel : la contrepartie dans les mesures envers les personnes sans emploi en Europe et en Amérique du Nord, Montréal, Presses de l'Université de Montréal.

ELLIOTT, M. (1996). «Impact of work, family, and welfare receipt on women's self-esteem in young adulthood », Social Psychology Quarterly, vol. 59, n 1, p. 80-95.

ENSMINGER, M. (1995). «Welfare and psychological distress: A longitudinal study of African American urban mothers », Journal of Health and Social Behavior, vol. 36, p. 346-359.

ESPING-ANDERSEN, G. (1999). Social Foundations of Postindustrial Economies, Oxford, Oxford University Press.

Esping-Andersen, G., D. Gallie, A. Hemerijck et J. Myles (2002). Why We Need a New Welfare State, Oxford, Oxford University Press.

Etzıoni, A. (1996). The New Golden Rule: Community and Morality in a Democratic Society, New York, Basic Books.

FAY, R. (1996). Enhancing the Effectiveness of Active Labour Market Policies: Evidence from Programme Evaluations in OECD Countries, Paris, OCDE.

FRANCE, A. (1998). «Why should we care?: Young people, citizenship and questions of social responsibility », Journal of Youth Studies, vol. 1, $n^{\circ} 1$, p. 97-111.

GADREY, J., et F. JANY-CATRICE (2003). Les indicateurs de richesse et de développement: un bilan international en vue d'une initiative française, Paris, ministère de l'Emploi, du Travail et de la Cohésion sociale.

GIDDENS, A. (2000). «La mondialisation, les inégalités et l'État d'investissement social », dans L. ARIZPE (coord.), Diversité culturelle, conflit et pluralisme, Paris, UNESCO, p. 63-70. 
GILBERT, N. (2002). Transformation of the Welfare State: The Silent Surrender of Public Responsibility, Oxford, Oxford University Press.

GIlbert, N., et R. VAN VoORHIS (dir.) (2001). Activating the Unemployed, New Brunswick/Londres, Transaction Publishers.

GUSTAFSSON, S., et F. STAFFORD (1995). « Links between early childhood programs and maternal employment in three countries », The Future of Children, vol. 5, $\mathrm{n}^{\circ} 3$, p. 161-174.

HAGEN, J., et L. DAVIS (1996). « Mothers' views on child care under the JOBS program and implications for welfare reform », Social Work Research, vol. 20, n 4, p. 263-273.

Hallsten, L., G. Grossı et H. WesterLund (1999). « Unemployment, labour market policy and health in Sweden during years of crisis in the 1990's ", International Archives of Occupational and Environmental Health, vol. 72, p. S28-S30.

Halvorsen, R., et P.H. Jensen (2004). « Activation in Scandinavian welfare policy: Denmark and Norway in a comparative perspective », European Societies, vol. 6, $n^{\circ}$ 4, p. 461-483.

HANDLER, J. F. (2003). « Social citizenship and workfare in the US and Western Europe: From status to contract », Journal of European Social Policy, vol. 13, $n^{\circ}$ 3, p. 229-243.

HASELL, M. J., et J. SCANZONI (1997). «Social networks and network-friendly housing in the U.S. », International Journal of Comparative Sociology, vol. 38, $n^{\text {os }} 3-4$, p. 289-296.

HeAly, T., et S. CôtÉ (2001). Du bien-être des nations : le rôle du capital humain et social, Paris, OCDE.

Jenson, J. (2004). Catching Up to Reality: Building the Case for a New Social Model, Ottawa, Canadian Policy Research Networks Inc.

KIRWAN, R., et L. MACFARLANE (1996). Stratégies pour le logement et l'intégration sociale dans les villes, Paris, OCDE.

Kleinman, M., et C. WhiteheAd (1999). « Housing and regeneration: The problem or the solution », National Institute Economic Review, vol. 170, p. 78-86.

LARSEN, J. E. (2001). The Active Society and Activation Policy. [www.ihis.aau.dk/gep/publicationer/nr4.pdf].

MARINETTO, M. (2003). «Who wants to be an active citizen? The politics and practice of community involvement », Sociology, vol. $37, \mathrm{n}^{\circ} 1$, p. 103-120.

MARTIN, J. (2000). Ce qui fonctionne dans les politiques actives du marché du travail: observations découlant de l'expérience des pays de l'OCDE, Paris, OCDE.

McANDREW, M., C. Tessier et G. BourgeAult (1997). « L'éducation à la citoyenneté en milieu scolaire au Canada, aux États-Unis et en France: des orientations aux réalisations, l'éducation comparée », Revue française de pédagogie, vol. 121, p. 57-77.

MCCLINTOCK, C., et S. LOWE (2001). «Welfare reform and its enduring questions: What have we learned from evaluation research? », dans G. JULNES et M. FOSTER (dir.), Outcomes of Welfare Reform for Families Who Leave TANF, San Francisco, Jossey-Bass, p. 9-20.

McGovern, M.A. (1998). «Early child care and education: Lessons from the French », Child \& Youth Care Forum, vol. 27, $\mathrm{n}^{\circ}$ 1, p. 21-37.

McKenNA, M., et D. WILLMS (1998). « Co-operation between families and schools: "What works" in Canada », Research Papers in Education, vol. 13, n 1, p. 19-41. 
MofFITT, R. (1992). «Incentive effects of the U.S. welfare system: A review », Journal of Economic Literature, vol. 30, $\mathrm{n}^{\circ}$ 1, p. 1-61.

OECD (1998). Key Employment Policy Challenges Faced by OECD Countries, Paris, OECD.

OORSCHOT, W. VAN (2004). «Balancing work and welfare: activation and flexicurity policies in The Netherlands, 1980-2000 », International Journal of Social Welfare, vol. 13, p. 15-27.

ORTHNER, D., et K. RANDOLPH (1999). «Welfare reform and high school dropout patterns for children », Children and Youth Services Review, vol. 21, n ${ }^{\circ \mathrm{s}}$ 9-10, p. 881-900.

Osberg, L., et A. ShARPE (2002). Une évaluation de l'indicateur du bien-être économique dans les pays de l'OCDE, Paris, ministère de l'Emploi, du Travail et de la Cohésion sociale.

Perret, B. (2002). Indicateurs sociaux: état des lieux et perspectives, Paris, Conseil de l'Emploi, des Revenus et de la Cohésion sociale (CERC).

Pierson, P. (1994). Dismantling the Welfare State? Reagan, Thatcher, and the Politics of Retrenchment, Cambridge, Cambridge University Press.

Prescott-Allen, R. (2003). Le bien-être des nations, Ottawa, Centre de recherches pour le développement international.

Ridgway, P., A. Simpson, F. Wittman et G. WheEler (1998). « Home making and community building: Notes on empowerment and place », dans B.L. LEVIN, A.K. BLANCH et A. JENNINGS (dir.), Women's Mental Health Services: A Public Health Perspective, Thousand Oaks, Sage Publications, p. 155-174.

RINGEN, S. (1997). Citizens, Families, and Reforms, Oxford, Clarendon Press.

Rose, N. (1999). Powers of Freedom: Reframing Political Thought, Cambridge, Cambridge University Press.

SEN, A. (1993). « Capability and well-being », dans M. Nussbaum et A. SEN (dir.), The Quality of Life, Oxford, Clarendon Press, p. 30-53.

SHIGEHARA, K. (1998). Préserver la prospérité dans une société vieillissante, Paris, OCDE.

SKEVIK, A. (2005). «Women's citizenship in the time of activation: The case of lone mothers in "needs-based" welfare states », Social Politics, vol. 12, n 1, p. 42-66.

SOMERVILLE, P. (1998). «Empowerment through residence », Housing Studies, vol. 13, $\mathrm{n}^{0} 2$, p. 233-257.

SomerVille, P., et A. Steele (1999). « Making oneself at home: The mediation of residential action », International Journal of Urban and Regional Research, vol. 23, n 1, 88-102.

SPENCE Boocock, S. (1995). «Early childhood programs in other nations: Goals and outcomes », The Future of Children, vol. $5, \mathrm{n}^{\circ} 3$.

ST. JOHN-BROOKS, C., et K. KELLEY-LAINÉ (1997). Les parents partenaires de l'école, Paris, OCDE.

St. Pierre, R., J. LAYzer et H. BARnes (1995). « Two-generation programs: Design, cost, and short-term effectiveness », The Future of Children, vol. $5, \mathrm{n}^{\circ} 3$.

TAYLOR-GoOBY, P. (2004). « Les nouveaux risques sociaux dans la société postindustrielle : les réactions d'Eurobaromètre aux politiques actives du marché du travail », Revue internationale de sécurité sociale, vol. 57, nº 3, p. 53-82. 
TREMBLAY, D. (2005). «Le principe de la "société active": comment affecte-t-il les modèles nationaux d'État social? », dans J.-L. BOUCHER et J.-Y. THÉRIAULT (dir.), Petites sociétés et minorités nationales, Sainte-Foy, Presses de l'Université du Québec, p. 305-315.

Tremblay, D., Y. Assogba, J.-L. Boucher et G.-C. Guindon (2002). Activation des politiques publiques et bien-être de la population: une recension des écrits, Gatineau, Chaire de recherche du Canada en développement des collectivités.

WALKER, R., et M. WISEMAN (2003). «Optimiser la protection sociale : les politiques d'activation dans le cadre du New Labour », Revue internationale de sécurité sociale, vol. 56, $\mathrm{n}^{\circ}$ 1, p. 3-34.

WAlters, W. (1997). « The "active society": New designs for social policy », Policy and Politics, vol. $25, n^{\circ} 3$, p. 221-234.

WARE, N. (1999). «Evolving consumer households: An experiment in community living for people with severe psychiatric disorders », Psychiatric Rehabilitation Journal, vol. 23, $\mathrm{n}^{\circ} 1$, p. 3-10.

WEICHER, J. (1996). «A new war on poverty: The Kemp Program to empower the poor », dans M.R. DARBY (dir.), Reducing Poverty in America: Views and Approaches, Thousand Oaks, Sage, p. 199-223.

WINTER, M. DE, C. BAERVELdT et J. KoOISTRA (1999). « Enabling children: Participation as a new perspective on child-health promotion », Child: Care, Health and Development, vol. $25, \mathrm{n}^{\circ} 1$, p. 15-25.

WUHL, S. (1996). Insertion : les politiques en crise, Paris, Presses universitaires de France.

ZAslow, M., E. Oldham, K. Moore et E. MAgenheim (1998). «Welfare families' use of early childhood care and education programs, and implications for their children's development », Early Childhood Research Quarterly, vol. 13, n 4, p. 535-563. 